\title{
Timing inconsistencies in the calculation of funds of funds net asset value
}

\author{
C. Louargant ${ }^{1}$, L. Neuberg ${ }^{2} \&$ V. Terraza ${ }^{3}$ \\ ${ }^{1}$ University of Metz, Grefige-Ceremo, France \\ ${ }^{2}$ Fortis Investments, Luxembourg \\ ${ }^{3} \mathrm{LSF}$, University of Luxembourg
}

\begin{abstract}
The purpose of this paper is to analyze the impact upon tracking errors of timing inconsistencies in the calculation of Funds of Funds (FoF) net asset value (NAV). We examine how these timing inconsistencies produce noise in the NAV of FoF and therefore noise in the tracking error. We construct Funds of Funds and calculate NAVs of these FoF using underlying NAVs at different dates. We then compare series of tracking errors to analyze the impact of the timing inconsistencies and formalize a relation adjusting the tracking error including the error term generated by these timing inconsistencies.
\end{abstract}

Keywords: Funds of Funds, NAV calculation, tracking error.

\section{Introduction}

A fund of funds is a mutual fund, which invests in other mutual funds. These funds were designed to achieve even greater diversification than traditional mutual funds. In the literature we have found many researches on funds but little on fund of funds. Nevertheless, the number of fund of funds increases each year and the proportion of this tool increases too since 2002. Identifying and selecting the most appropriate fund to use in an investor's portfolio are the major aspects of investment strategies. Two of the most important quantitative measures traditionally used are tracking error (TE) and the information ratio (IR). When used properly, these tools give interesting information to make decisions. However, one important problem recently advocated in the literature [1, 2], concerns the difficulty to estimate TE. We compute annualised tracking errors for 1700 Funds and 280 Funds of Funds. We compare the two non Gaussian 
distributions of TE using Kolmogorov Smirnov and Wilcoxon-Mann Whitney tests. The results of tests reveal that the two sample of TE don't follow the same distribution. In this paper, we consider another source of bias to estimate TE relative to the analysis of fund of funds: the excess volatility of Tracking Errors due of time inconsistencies. Although the problem of non-synchronous data has been already shown in mutual funds by previous studies [3-7], it has found more pronounced in fund of funds due to specifics microstructure effects in fund of fund markets. We show that time inconsistencies in the NAV of FOF create autocorrelation in series and induced a biased indicator of risk. For that we introduce a correction term in the TE formula. Our analysis proceeds in the following steps: section 2 describes the construction of fund of funds. Section 3 presents the simulation results of impact upon tracking errors of timing inconsistencies in the calculation of FoFs NAV. We compare the return series of a FoF with the return series of its benchmark, and show the problem due to the timing inconsistencies in the calculation. We examine how these timing inconsistencies produce noise in the NAV and therefore noise in the tracking error. In section 4, we analyze the autocorrelation of simulated FoF and propose a correction term in TE formula. In section 5 we conclude.

Table 1: $\quad$ The construction of funds of funds.

\begin{tabular}{|clc|}
\hline & \multicolumn{1}{c}{ Name } & Initial Weight \\
1 & Schroder ISF Japanese Equity C Acc & $5 \%$ \\
2 & Pictet F-Emerging Markets-P & $5 \%$ \\
3 & Pioneer Funds Top European Players A No Dis EUR & $5 \%$ \\
4 & Ofima Cible & $5 \%$ \\
5 & JPMF Europe Strategic Value A EUR & $5 \%$ \\
6 & MLIIF US Focused Value A2 USD & $5 \%$ \\
7 & Vanguard US Opportunities Institutional USD & $5 \%$ \\
8 & Templeton Euroland A Acc & $5 \%$ \\
9 & SGAM Fund Equities US Concentrated Core B & $5 \%$ \\
10 & SGAM Fund Equities US Relative Value A & $5 \%$ \\
11 & Fidelity Funds - European Aggressive Fund & $5 \%$ \\
12 & CA Funds Emerging Markets I Cap (USD) & $5 \%$ \\
13 & ACM Bernstein-European Value Portfolio A EUR & $5 \%$ \\
14 & AXA Rosenberg Eurobloc Equity Alpha A EUR & $5 \%$ \\
15 & Fidelity Funds - Japan Fund & $5 \%$ \\
16 & Henderson HF Pan European Equity A2 & $5 \%$ \\
17 & INVESCO GT Pan European A & $5 \%$ \\
18 & Gartmore CS Eurobloc & $5 \%$ \\
19 & Franklin US Equity A Acc USD & $5 \%$ \\
20 & GAM Star European Equity EUR Accumulation Class & $5 \%$ \\
\hline
\end{tabular}

\section{Construction of funds of funds}

The first stage in our FoF construction concerns the benchmark definition. As the objective of the study is to analyze the impact of timing inconsistencies on the FoF' TE, an interesting benchmark has to incorporate the larger possible range of international markets in order to maximize the overlapping effect induced by time zones. We choice the following benchmark with the proportion 
in the brackets: MSCI EMU (20\%), MSCI Europe ex-EMU (20\%), MSCI USA (20\%), MSCI Japan (20\%), MSCI Emerging Markets Free (20\%). The second stage is the construction of the FoF itself. We dispose of every NAV of 20 underlying funds for every market day during the period of May 2002 to March 2005. These funds are equally weighted, and we keep the number of parts of underlying funds unchanged during time without any buy and sell, avoiding by the way transactions costs. These data comes from the Lipper Database. Our simulated FoF is presented in table 1.

\section{Impact upon tracking errors of timing inconsistencies in the calculation of FoFs NAV}

\subsection{Timing inconsistencies}

When the NAV of a FoF is calculated, the NAVs of underlying funds are not always available at the same market date. This inconsistency is minimum (even null) when we dispose of all underlying NAVs at the same market day and maximum when we have only fifty percents of the available NAVs at one market day and fifty percents at another market day. At the optimal situation, the most recent underlying NAVs are available for the day before the calculation day. This is usually the case when the FoF has a benchmark defined on a single market. Nevertheless, every manager of FoF compare the return series of his FoF with the benchmark return series delayed by one day (the day before). This can be due to the time of publication of the underlying funds NAVs and also due to the fact that the funds relate to different markets with different closing times. The NAV of the FoF are therefore calculated using diverse market days. In practise, every manager of FoF compares the return series of his FoF at time $t$ with the benchmark return series delayed by one day (at t-1). This strategy creates timing inconsistencies. A solution should be to use all NAVs available two days before $(\mathrm{t}-2)$ in place of some in t-1 and some in t-2. Nevertheless, managers don't use this solution because legal rules avoid arbitrage opportunity that can be generated by Late Trading or Market Timing.

\subsection{The tracking error ratio measure}

Although there are a great number of risk measurement frameworks, the focus of the paper is the market risk of fund of funds relative to its benchmark. Tracking error (TE) is a commonly used summary statistical measure of relative risk to provide an acceptable range of relative performance. TE was first defined by Tobe [8] as the percentage difference between the portfolio (in our application the FOF) and its benchmark index the fund was designed to replicate. TE is estimated as the annualized standard deviation of the difference in returns. For investment funds, it represents the percentage change in the Net Asset Value (NAV) for each day of the whole time period required:

Percentage change in the NAV $=\frac{N A V \text { onday }(t)-N A V \text { on day }(t-1)}{N A V \text { onday }(t-1)}$ 
Mathematically, the tracking error (TE) is:

$$
\mathrm{TE}=\sqrt{\frac{\sum_{t=1}^{n}\left(R_{F O F}-R_{B}\right)^{2}}{N-1}}
$$

where $\mathrm{R}_{\mathrm{FOF}}$ is the return of FOF, $\mathrm{R}_{\mathrm{B}}$ the return of the benchmark and $\mathrm{N}$ the number of return periods

The annualized TE for daily observations is:

$$
\mathrm{TE}^{*} \sqrt{250}
$$

Lower the TE, closer are the returns of the fund to that of the benchmark.

\subsection{Impact upon relative risk measures of timing inconsistencies}

Lets assume that we calculate the NAV of our FoF at time t. At this time, the underlying funds haven't yet published their own NAV. We create fictive situations starting from an optimal situation on which we dispose of every underlying NAV at time $\mathrm{t}-1$ to the worst situation where the all underlying NAV are available only on time $\mathrm{t}-2$. Between these two extreme situations, we have NAV available on both $\mathrm{t}-1$ and $\mathrm{t}-2$. As we have 20 underlying funds, we create 21 simulations. Table 2 describes the simulation procedure. For each simulation, we compute the returns series on a daily basis, the annualised tracking errors (from the benchmark return series delay by one day), the beta, the correlation and finally the total risk (the volatility). Table 2 shows the influence on timing inconsistencies on measures of risk portfolio.

Table 2: $\quad$ Simulations results.

\begin{tabular}{|c|c|c|c|c|c|c|}
\hline Simulation & $\begin{array}{c}\text { Number of } \\
\text { NAV in T-1 } \\
\text { (in \%) }\end{array}$ & $\begin{array}{c}\text { Number of } \\
\text { NAV in T-2 } \\
\text { (in \%) }\end{array}$ & Tracking Error & Beta & Correlation & Volatility \\
\hline 1 & 100 & 0 & $5.88 \%$ & 0.90 & 0.93 & $15.27 \%$ \\
\hline 2 & 95 & 5 & $6.19 \%$ & 0.87 & 0.92 & $14.80 \%$ \\
\hline 3 & 90 & 10 & $6.22 \%$ & 0.83 & 0.92 & $14.18 \%$ \\
\hline 4 & 85 & 15 & $6.69 \%$ & 0.78 & 0.91 & $13.62 \%$ \\
\hline 5 & 80 & 20 & $7.32 \%$ & 0.74 & 0.89 & $13.18 \%$ \\
\hline 6 & 75 & 25 & $8.01 \%$ & 0.70 & 0.86 & $12.81 \%$ \\
\hline 7 & 70 & 30 & $8.81 \%$ & 0.67 & 0.83 & $12.64 \%$ \\
\hline 8 & 65 & 35 & $9.88 \%$ & 0.63 & 0.78 & $12.78 \%$ \\
\hline 9 & 60 & 40 & $10.94 \%$ & 0.60 & 0.73 & $13.01 \%$ \\
\hline 10 & 55 & 45 & $11.48 \%$ & 0.55 & 0.69 & $12.60 \%$ \\
\hline 11 & 50 & 50 & $12.51 \%$ & 0.53 & 0.64 & $13.00 \%$ \\
\hline 12 & 45 & 55 & $13.42 \%$ & 0.48 & 0.58 & $13.11 \%$ \\
\hline 13 & 40 & 60 & $14.39 \%$ & 0.45 & 0.52 & $13.42 \%$ \\
\hline 14 & 35 & 65 & $14.54 \%$ & 0.42 & 0.50 & $13.08 \%$ \\
\hline 15 & 30 & 70 & $15.20 \%$ & 0.38 & 0.46 & $13.11 \%$ \\
\hline 16 & 25 & 75 & $15.75 \%$ & 0.34 & 0.41 & $13.07 \%$ \\
\hline 17 & 20 & 80 & $16.76 \%$ & 0.31 & 0.36 & $13.76 \%$ \\
\hline 18 & 15 & 85 & $17.66 \%$ & 0.26 & 0.30 & $13.98 \%$ \\
\hline 19 & 10 & 90 & $18.47 \%$ & 0.23 & 0.25 & $14.44 \%$ \\
\hline 20 & 5 & 95 & $19.07 \%$ & 0.20 & 0.21 & $14.64 \%$ \\
\hline 21 & 0 & 100 & $20.01 \%$ & 0.16 & 0.17 & $15.28 \%$ \\
\hline
\end{tabular}


We can observe on figures 1 and 2, linear relationships between them: the evolution of the TE is positively linear while the evolution of the beta is negatively linear.

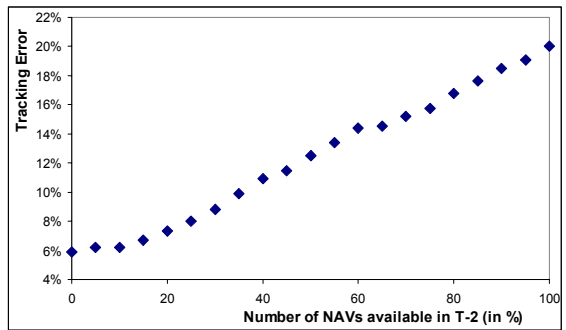

Figure 1: Influence on timing inconsistencies on tracking error.

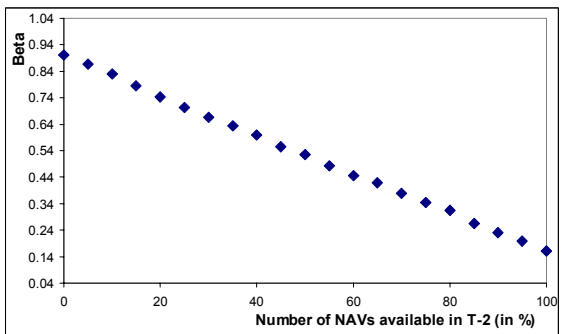

Figure 2: $\quad$ Influence on timing inconsistencies on beta.

Indeed, the higher is the correlation between the benchmark and the FoF and the lower is the tracking error. As timing inconsistencies increase (100\% of NAV available in $\mathrm{t}-2$ for the worst case), the correlation between the benchmark and the FoF falls and the TE increases exponentially.

To verify if the outcome of simulations is correlative to the choice of the benchmark, we compute the same simulations using different benchmarks and we obtain the same kinds of behaviours in regards of time inconsistencies.

But due to our diversified benchmark, which maximises the overlapping effect induced by time zones, we observe the maximum timing inconsistency effect in computing TE, beta and correlation for our simulations.

\section{Linear filtering and the "adjusted" tracking error}

The next step in the analysis is the modeling of the linear dependencies within the funds of funds data sets. Indeed, time inconsistencies can create 
autocorrelation in series and then can explain one part of excess volatility in tracking error.

This phenomenon has already been observed in mutual funds. However, the goals of this step are to determine the effects, if any, that such linear pre-filtering has on tracking error index.

We apply the Ljung Box statistics to test the autocorrelation in simulation, in $250 \mathrm{FoF}$ and 250 funds. The results of the tests on real series reveal that statistics is more often significant for FoF than funds.

The Ljung Box statistics reveal the presence of autocorrelation both in our simulations and the benchmark.

To take into account the autocorrelation effect, we pre-filter the original returns. This series is in effect an integrated series. More specifically, it follows an $\mathrm{AR}(1)$ or an $\mathrm{AR}(2)$ process. Thus, the elements of the processes must next be filtered out before the final, proxy series of returns could be obtained.

For the benchmark, the I(1) elements is removed by taking the first differences within the series, leaving the following:

$$
R_{B t}-0.1472 R_{B_{t-1}}=\eta_{t}
$$

For our fund of funds series, the $\mathrm{I}(1)$ or $\mathrm{I}(2)$ elements is removed using the following regressions:

$$
R_{F O F_{t}}-\rho_{1} R_{F O F_{t-1}}-\rho_{2} R_{F O F_{t-2}}=\varepsilon_{t}
$$

where the parameters estimation of equation 5 is given in table 3 .

It is the series of residuals from the models (4) and (5), which are $\hat{\eta}_{t}=R_{B}^{\text {Proxy }}$ and $\hat{\varepsilon}_{t}=R_{F O F}^{\text {Proxy }}$ that finally serves as the proxy series for returns.

The next step is to define an "adjusted" Tracking Error from proxy returns of funds of funds:

$$
\text { Adjusted TE }=\sqrt{\frac{\sum_{t=1}^{n}\left(\hat{\varepsilon}_{t}-\hat{\eta}_{t}\right)^{2}}{N-1}}
$$

This indicator is based on a linear pre-filtering approach to estimate the tracking error ratio. In comparison with the traditional TE statistics, the adjusted tracking error pre-filter the original returns in order to avoid linear dependencies. The following picture compares the two indicators. 
Table 3: $\quad$ Regression estimations.

\begin{tabular}{|c|c|c|c|c|}
\hline Simulations & \multicolumn{2}{|c|}{$\hat{\rho}_{1}$} & \multicolumn{2}{|c|}{$\hat{\rho}_{2}$} \\
\hline 1 & 0.2488 & $(0.0358)$ & & 1 \\
\hline 2 & 0.2739 & $(0.0356)$ & & / \\
\hline 3 & 0.3223 & $(0.035)$ & & / \\
\hline 4 & 0.3496 & $(0.0346)$ & & / \\
\hline 5 & 0.3746 & $(0.0343)$ & & / \\
\hline 6 & 0.4898 & $(0.0366)$ & -0.1338 & $(0.0366)$ \\
\hline 7 & 0.4892 & $(0.0366)$ & -0.1416 & $(0.0366)$ \\
\hline 8 & 0.4709 & $(0.0366)$ & -0.1314 & $(0.0366)$ \\
\hline 9 & 0.4709 & $(0.0366)$ & -0.1314 & $(0.0366)$ \\
\hline 10 & 0.5132 & $(0.0365)$ & -0.159 & $(0.0365)$ \\
\hline 11 & 0.522 & $(0.0364)$ & -0.1651 & $(0.0364)$ \\
\hline 12 & 0.6245 & $(0.0359)$ & -0.2337 & $(0.0359)$ \\
\hline 13 & 0.5957 & $(0.0361)$ & -0.2099 & $(0.0361)$ \\
\hline 14 & 0.6346 & (0.036) & -0.2258 & $(0.036)$ \\
\hline 15 & 0.6319 & $(0.0362)$ & -0.2039 & $(0.0362)$ \\
\hline 16 & 0.5374 & $(0.0367)$ & -0.1145 & $(0.0367)$ \\
\hline 17 & 0.4994 & $(0.0367)$ & -0.1013 & $(0.0367)$ \\
\hline 18 & 0.4021 & $(0.0367)$ & & \\
\hline 19 & 0.4354 & $(0.0368)$ & -0.0833 & $(0.0368)$ \\
\hline 20 & 0.3303 & $(0.0348)$ & & \\
\hline 21 & 0.299 & $(0.0352)$ & & \\
\hline
\end{tabular}

(.) standard deviations

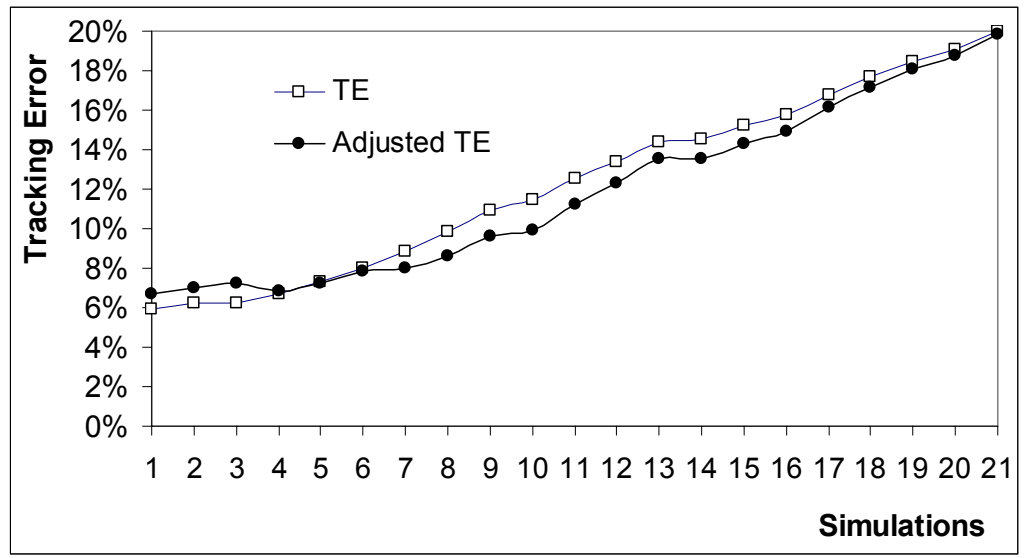

Figure 3: $\quad$ Comparison between TE and Adjusted TE. 
We can observe a substantial improve of our results from figure 3: the correction of autocorrelation gives a better estimation of the Tracking Error. However, linear filtering is not sufficient. Indeed, even if the effect of time inconsistencies can be reduced by taking account autocorrelation of the series it seems that it's cannot explain the excess of volatility observed in tracking error of funds of funds. Some others sources can be due to statistical properties like residual non-linear dependencies, or perhaps in the construction on funds of funds itself.

\section{Conclusion}

We analyzed another source of bias in the calculation of tracking error: time inconsistencies. We concluded that these inconsistencies create excess volatility in Fund of Funds tracking error. Moreover, we showed that time inconsistencies in the NAV of Fund of Funds create autocorrelation in series and so induced a biased indicator of risk. We construct an adjusted tracking error formula estimated from autoregressive processes of returns. Our principal result is that taking into account the autocorrelation effect in NAV of funds of funds improves our risk estimation. The next step will be to ameliorate the quality of the correction of Tracking error formula by improving the analysis of the residuals terms.

\section{References}

[1] Clarke R.G., Krase S. and Statman M., Tracking errors, Regret and tactical asset allocation, The Journal of Portfolio Management, Spring, pp.16-24, 1994.

[2] Rudolf M., Wolter H and Zimmermann H., A linear model for tracking error minimization, The Journal of Banking and Finance, 23, pp.85-103, 1999.

[3] French and Roll, Stock return variances: The arrival of information and the reaction of traders Journal of Financial Economics ,17, pp.5-26, 1986.

[4] Kadlec G.B. and Patterson D.M., A transaction data analysis of nonsynchronous trading, Review of Financial Studies, fall, 12 (3), pp.609630, 1999.

[5] Goetzmann W.N., Ivkovich Z. and Rouwenhorst K.G., Day trading international mutual funds: evidence and policy solutions, working paper ICF00-03, Yale School of Management, Yale University, 2000.

[6] Chalmers J.M.R., Edelen R.M. and Kadlec G.B., Predictable changes in NAV: the Wildcard option in transacting mutual-fund shares, working paper 08-00, Wharton School, University of Pennsylvania, 2000.

[7] Ammann M. and Zimmermann H., Tracking error and tactical asset allocation, Financial Analysts Journal, 57 (2) March/April, pp.32-43, 2001.

[8] Tobe C, Tracking error: An essential tool in evaluating index Funds, the Journal of Investment Consulting, November, pp.8-11, 1999. 